\title{
BMJ Open Effect of RUSI-based core stability exercise on chronic non-specific low back pain patients: study protocol for a randomised controlled trial
}

\author{
Shanshan Lin (D) ,1,2 Bo Zhu, ${ }^{3}$ Yiyi Zheng, ${ }^{2}$ Shufeng Liu, ${ }^{2}$ Chuhuai Wang ${ }^{2}$
}

To cite: Lin S, Zhu B, Zheng Y, et al. Effect of RUSI-based core stability exercise on chronic non-specific low back pain patients: study protocol for a randomised controlled trial. BMJ Open 2021;11:e047317. doi:10.1136/ bmjopen-2020-047317

- Prepublication history for this paper is available online. To view these files, please visit the journal online (http://dx.doi. org/10.1136/bmjopen-2020047317).

SL and BZ contributed equally.

$\mathrm{SL}$ and $\mathrm{BZ}$ are joint first authors.

Received 25 November 2020 Accepted 29 0ctober 2021

Check for updates

(C) Author(s) (or their employer(s)) 2021. Re-use permitted under CC BY-NC. No commercial re-use. See rights and permissions. Published by BMJ.

${ }^{1}$ Department of Rehabilitation Medicine, Jiangmen Central Hospital, Jiangmen, Guangdong, China

${ }^{2}$ Department of Rehabilitation

Medicine, Sun Yat-sen University First Affiliated Hospital,

Guangzhou, Guangdong, China

${ }^{3}$ Department of Hepatobiliary

Surgery, Jiangmen Central

Hospital, Jiangmen, Guangdong, China

Correspondence to

Professor Chuhuai Wang;

wangchuh@mail.sysu.edu.cn

\section{ABSTRACT}

Introduction Low back pain (LBP) is one of the most highly prevalent pain both in developed countries and lowincome and middle-income countries. Despite increasing healthcare resources and numerous treatment methods for LBP, the efficacy of these therapeutic strategies is still uncertain. Recently, core stability exercise (CSE) is popularly applied as a preventive or rehabilitative method in the treatment of LBP. However, the adequate activation of the local muscle systems of CSE needs further optimisation and quantification. This trial aims to investigate the feasibility and efficacy of CSE monitored by real-time ultrasound image (RUSI) on LBP individuals. Methods and analysis Forty subjects with chronic non-specific LBP (CNLBP), aged from 20 to 50 years, will be randomly allocated into two groups using sealed, consecutively numbered opaque envelopes: (1) study group (SG): CSE monitored by RUSI and (2) control group (CG): identical CSE without monitoring. Interventions will last 30 mins, two times a week for 8 weeks. The primary outcomes include pain intensity, disability and quality of life, and the secondary outcomes will be the postural control static stability, onset timing of trunk muscles activation, ultrasound images of muscle thickness and surface electromyography (sEMG) signal of muscle activities. Outcome measures will be collected at baseline, 4 and 8 weeks during training, and at 6 months follow-up. Data will be collected and analysed by an assessor blinded to group allocation. Effect sizes and mixed-model repeated measures analysis of variance ( 2 groups $\times 4$ time points) will be calculated.

Ethics and dissemination This protocol and informed consent has been approved by the Institutional Research Ethics Committee of the First Affiliated Hospital, Sun Yat-sen University (Approval number: [2020] 254-1). The findings of this study will be disseminated to participants through social networks and will be submitted to peerreviewed journals and scientific conferences.

Trial registration number Chinese Clinical Trial Registry (ChiCTR2000034498).

\section{INTRODUCTION}

Non-specific low back pain (LBP) is one of the most common musculoskeletal pain over the world and affects $85 \%-90 \%$ of the population at some point in their life. ${ }^{12}$ LBP causes a
Strengths and limitations of this study

- This randomised controlled trial is the first attempt to systematically investigate the efficiency of the real-time ultrasound image-based core stability exercise in individuals with chronic non-specific low back pain (CNLBP).

- This study will show the effect of short-term and medium-term applications of core stability exercise (CSE) combined with real-time biofeedback in individuals with CNLBP, which could be a more effective method to treat LBP patients.

- Outcome measures include various aspects of LBP problems (pain intensity, functional disability, quality of life and quantitative indicators such as electromyography, ultrasound, etc).

- There is no standardised intervention protocol for use of CSE therapy in LBP subjects.

- Restriction to the age range of participants may limit the generalisability of the findings.

significant burden on social economy, which is related to increased direct (eg, medical care) and indirect costs (such as sick leave and social productivity). ${ }^{34}$ Therefore, finding effective treatments for chronic non-specific LBP (CNLBP) is a major challenge for clinicians and researchers, and of great importance for patients with LBP.

Various rehabilitation and treatment methods including electrical stimulation, massage, acupuncture and motor control training have been used to the management of LBP patients. ${ }^{5-8}$ However, the effectiveness of these treatment programmes is still questionable and uncertain. In the last few years, core stability exercise (CSE) has been reported to be important in the management of LBP, not only for the prevention and reduction back pain and disability but also for improving spinal stability and flexibility. ${ }^{9}$

Core stability is defined as the ability to maintain a stable neutral zone of the spine. According to the function and activity, the 
core muscles can be categorised into two main groups. The global or superficial muscles are the primer movers of the trunk. The local or deep muscles provide a stiffening effect on the lumbar spine through attaching to the thoracolumbar fascia and play important roles in the segmental stability. ${ }^{10}{ }^{11}$ However, patients with LBP demonstrate defection in these local muscles, such as atrophy, fat infiltration and preferential activation delay which makes anticipatory postural adjustment (APA) difficult and eventually result in damage to the spine. ${ }^{12-14}$

Previous studies have reported that CSE improves spinal stability and decreases pain for individuals with lumbar instability in terms of neuromuscular control ${ }^{1516}$ However, the application of CSE targeting transverse abdominis (TrA) and lumbar multifidus (LM) needs further exploration and optimisation due to the activation of local muscles that appear to be particularly difficult in LBP individuals, and also because of the difficulty to feel or palpate of the deep muscles. Studies have previously evaluated the effect of trunk stabilisation merely investigated deep muscle thickness using ultrasound or examined the changes in muscle activity using electromyography (EMG) in older or healthy subjects, ${ }^{17-20}$ but there were few studies reported the efficiency of real-time ultrasound image (RUSI)-based CSE on LBP patients. Therefore, reliable, sensitive and non-invasive measurements are important to provide real-time and precise information of trunk muscles on specific functional tasks during CSE. ${ }^{1221}$ Thus, real-time display of deep muscle thickness changes monitored by ultrasound during functional re-education and training would be helpful to provide biofeedback and instruction to the therapists and patients. ${ }^{22} 23$

Based on the above background information, RUSI will be used to provide real-time biofeedback of observing the deep muscles morphological changes in this study. We hypothesise that, in contrast to the general CSE group, the study group (SG) monitored by RUSI will induce greater activities of TrA, and more effectively promoting trunk stability, reducing pain, and improving disability.

\section{METHODS}

\section{Study design}

To evaluate the effects of CSE monitored by RUSI on the effectiveness of LBP, a prospective two-arm randomised controlled trial will be conducted. This study will take place in an outpatient rehabilitation centre in the first affiliated hospital of Sun Yat-sen University. Participants with CNLBP will be randomly allocated into one of two groups: (a) SG: CSE monitored by RUSI to increase subjects' activity levels and (b) control group (CG): identical CSE without monitoring. All of the participants in the two groups will be treated with specified CSEs for 30 min per session, two sessions per week for up to 8 weeks. This study protocol follows the guidelines described in the 'Standard Protocol Items: Recommendations for Interventional Trials' statement. ${ }^{24}$

\section{Approval and registration of the study}

The study design, procedures and informed consent were approved by the Institutional Research Ethics Committee of the First Affiliated Hospital, Sun Yat-sen University (Approval number: [2020] 254-1) and was prospectively registered in the Chinese Clinical Trial Registry. We confirm that all methods are performed following the ethical standards of the Declaration of Helsinki. The subjects will be informed about the objectives and procedures of the study. When eligible for inclusion, they will be requested to sign the informed consent form.

\section{Participant involvement}

All of the participants will not be involved in the design, conduct and dissemination plans of our research. The personal data of the participants such as name, age, body mass and so on will be numerically coded and stored in a database, which can only be accessed by the researcher responsible for the randomisation and blinding process during the study. Individual evaluation results will be available to participants on completion of the trial.

\section{Eligibility criteria}

A total of 40 patients will be recruited in this study via the outpatient department of the First Affiliated Hospital, Sun Yat-sen University, flyers, and social media sites. All personal data we collected will be confidential. Inclusion criteria for the study are (a) male and female, ages between 20 and 50 years; (b) LBP for at least 3 months, but no radiculopathy, specific spinal disease, or nerve root pain $^{25}$; (c) the scores of visual analogue scale (VAS) range from 3 to 7 and (d) body mass index (BMI) within $\pm 10 \%$ of international standards. Exclusion criteria are a history of abdominal or spinal surgery; previous experience of ultrasound imaging evaluation on trunk muscles; comorbid health conditions that would disturb active participation in the training programmes (eg, ankylosing spondylitis, rheumatoid arthritis, fractures, tumours, systemic disease, severe neurological and psychological disorders), or pregnancy.

\section{Sample size calculation}

The sample size was defined according to the previous research results reported by Zheng et al. ${ }^{23}$ The sample size calculation for this study is performed based on the change of the TrA activation ratio at 8-week treatment and assuming $80 \%$ power and $5 \%$ of significance to detect a significant difference of the TrA activation ratio among groups at 0.1 points, and the SD of 0.05 points. A minimal sample size of 33 patients is consequently to be included in the analysis. As a dropout rate of $20 \%$ is assumed, a total of 40 participants will be included in the study (20 in the SG, 20 in the CG).

\section{Randomisation and blinding}

Immediately after inclusion and baseline assessment, subjects will be randomised into one of the two groups by an investigator who is not involved in the recruitment or other study procedures of the patients. The randomisation 


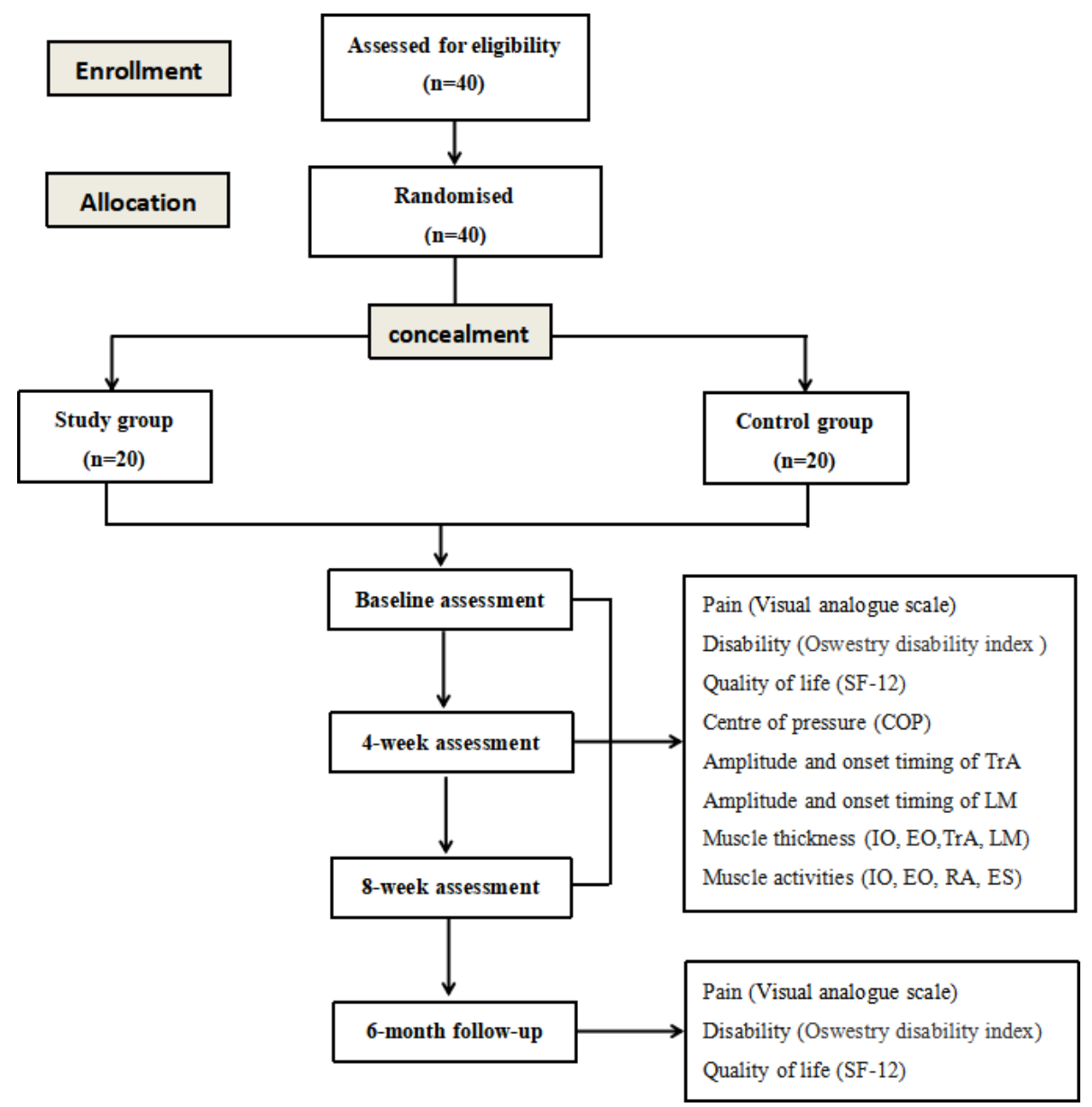

Figure 1 Flow diagram of the study. CSE, core stability exercise; EO, external oblique; IO, internal oblique; LM, lumbar multifidus; RA, rectus abdominis; SF-12, 12-Item Short-Form Health Survey; TrA, transverse abdominis; ES, erector spinae.

sequence is generated relying on a computer-generated randomization schedule (www.randomization.com). The allocation of subjects will be concealed by using consecutive numbered, sealed and opaque envelopes. ${ }^{26}$ The study assessor will be blinded to the allocated groups of the patients. However, it will not be possible for the therapist or the patients to be blinded. The flow of the study is summarised in figure 1 .

\section{Interventions}

All participants will be randomly allocated into one of two groups (all of the subjects will receive the same CSE training with or without real-time biofeedback). Interventions will last 30 min per session and will happen two times a week for 8 weeks. The therapist will demonstrate and explain a modified CSE training plan (shown in table 1) to the subjects in the two groups based on the previous studies. ${ }^{2327}$ They will also be instructed not to initiate any intervention while during the study. In the first 4 weeks, the participants will be asked to finish primary training, and in the next 4 weeks, they will be required to finish superior training using a sling setting. During training, RUSI will be used to provide real-time feedback in the SG to guarantee the correct contraction of the trunk muscles. The real-time biofeedback using RUSI will be maintained throughout the whole training process. The activities of the superficial muscles, such as RA, are not allowed to be more intense than those of the deep muscles namely TrA during exercise. The protocol of CSE training programmes is shown in table 1 and the placement of the EMG electrode and the ultrasound transducer for realtime biofeedback and examination are shown in table 2 concerning the recommendation by SENIAM and the method applied in previous studies. ${ }^{23} 28-30$

\section{Data collection}

Data collection will occur at baseline, 4, 8 weeks and 6 months follow-up. During training, all data will be recorded and saved into the trial database. Participants will also be asked to complete follow-up over the telephone by one of the researchers. If participants do not complete the evaluation within 2 days of the scheduled date, they will receive a message reminder or will be contacted by one of the study researchers.

\section{Primary outcome measures}

Pain

Self-reported pain will be investigated using the VAS. The VAS is a tool with a $10 \mathrm{~cm}$ ruler and the participants will be asked to rate their pain intensity by moving the marker 


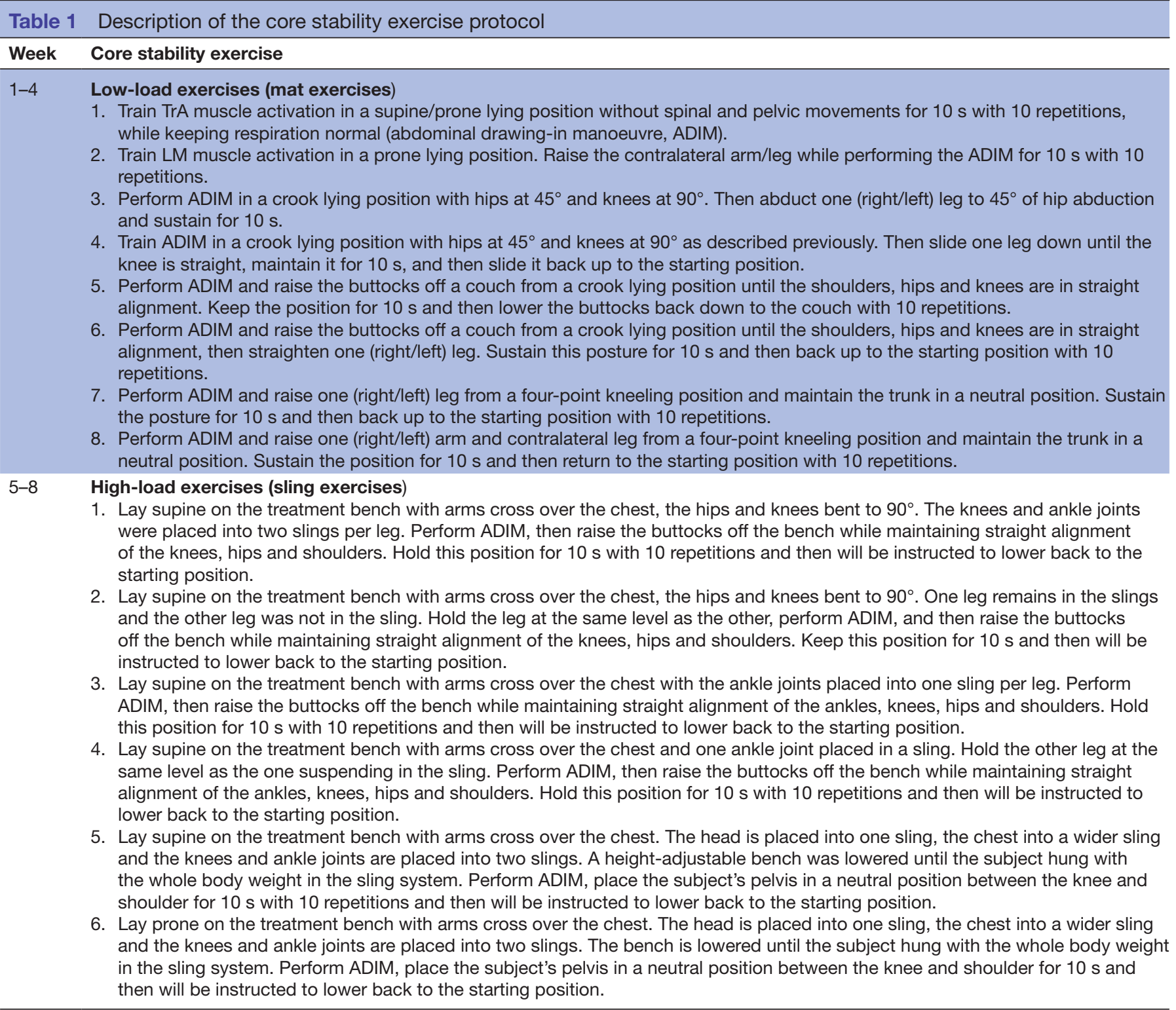

LM, lumbar multifidus; TrA, transversus abdominis.

indicating his or her intensity of pain. The VAS was horizontally positioned with the extremes labelled 'least possible pain' and 'worst possible pain'. ${ }^{31}$ VAS will be assessed in all-time points (ie, baseline, 4 weeks, 8 weeks and 6 months follow-up).

\section{Disability}

The Oswestry disability index (ODI) is a scale containing 10 sections, each section rated on a $0-5$ scale, the greater number represents greater disability. Relative values will be shown as total score/total possible score $\times 100 \%{ }^{31}$ Participants will be asked to select the items that describe the degree of disability on the day of the evaluation.

\section{Health-related quality of life}

The quality of life is evaluated by the 12-Item ShortForm Health Survey (SF-12). ${ }^{32} 33$ SF-12 consists of eight health scales namely physical or social functioning, role limitations due to physical or emotional problems, bodily pain, general health, vitality and perceived mental health. Raw scores in the eight domains are combined transformed into physical and mental health composite scores, which both range from 0 (lowest) to 100 (highest). Higher scores represent better health and well-being.

\section{Secondary outcome measures}

Postural control: static stability

The centre of pressure (COP) sways parameters will be evaluated on a Balancing Instrument (Prokin 254P, TecnoBody Company, Italy) to assess the balance performance during quiet standing. ${ }^{34-36}$ To assess postural stability in bipedal stance, the individuals will be asked to stand statically in a standardised position on the platform with their arms at their sides and eyes (open or closed) looking straight at the computer screen. To assess static 
Table 2 Placement of the electromyography electrodes and ultrasound transducer

\begin{tabular}{|c|c|}
\hline Muscle & Electrode placement location \\
\hline $\begin{array}{l}\text { Transversus } \\
\text { abdominis/ } \\
\text { internal oblique } \\
\text { (TrA/IO) }\end{array}$ & $\begin{array}{l}\text { Along either side of the course of the } \\
\text { underlying muscle fibres and centred } 2 \\
\mathrm{~cm} \text { cephalic to the pubic bone, just lateral } \\
\text { to the midline, and parallel to the superior } \\
\text { pubic ramus }\end{array}$ \\
\hline $\begin{array}{l}\text { External oblique } \\
\text { (EO) }\end{array}$ & $\begin{array}{l}\text { Halfway between the iliac crest and the } \\
\text { twelfth rib at a slightly oblique angle }\end{array}$ \\
\hline $\begin{array}{l}\text { Rectus } \\
\text { abdominis (RA) }\end{array}$ & $2 \mathrm{~cm}$ lateral to the umbilicus \\
\hline $\begin{array}{l}\text { Erector spinae } \\
\text { (ES) }\end{array}$ & $2-3 \mathrm{~cm}$ lateral to the $\mathrm{L} 3$ level \\
\hline $\begin{array}{l}\text { Lumbar } \\
\text { multifidus (LM) }\end{array}$ & $\begin{array}{l}\text { A line from posterior superior iliac spine to } \\
\text { the spinous process interval between } L 1 \\
\text { and } L 2 \text {, at the level of } L 5 \text { spinous process } \\
\text { (about } 2 \mathrm{~cm} \text { from the posterior midline) }\end{array}$ \\
\hline Muscle & $\begin{array}{l}\text { Ultrasound transducer placement } \\
\text { location }\end{array}$ \\
\hline TrA/IO/EO & $\begin{array}{l}\text { Along the midaxillary line at the level of } \\
\text { the umbilicus }\end{array}$ \\
\hline LM & $2 \mathrm{~cm}$ lateral to the $\mathrm{L} 4$ spinous process \\
\hline
\end{tabular}

stability in a monopedal stance, subjects will be asked to stand on one leg while the other leg is flexed off the floor with their arms crossover the chest. All tests will maintain $30 \mathrm{~s}$ with two repetitions. During the measurement, the COP sway area $\left(\mathrm{mm}^{2}\right)$ and sway length $(\mathrm{mm})$ will be recorded to evaluate the static balance and stability.

\section{Postural control: trunk position sense}

Trunk position sense, represented by trunk reposition errors (TRE), will be evaluated with a digital inclinometer (Prokin 254P, TecnoBody Company, Italy). The subjects will be asked to stand upright in a relatively standardised position. Then the inclinometer will be placed at the level of $\mathrm{T} 4$, the subjects will be asked to flex the trunk approximately $30^{\circ}$ in the sagittal plane and hold this position for $3 \mathrm{~s}$ to remember the position sense, then back to the starting position with two repetitions. After the initial trials, they will be asked to duplicate the previously attained position in the eye-closed condition three times and hold the position for $3 \mathrm{~s}$ each time. The absolute differences between the original position and the other three attempts will be calculated, and the mean of the three calculated data will be adopted to assess the trunk position sense. $^{37} 38$

\section{Muscle activity recorded by EMG}

Trunk muscle activity will be recorded using wireless surface EMG sensors (Trigno Wireless EMG system, Delsys, Boston, USA). Careful skin preparation will be performed to increase electrode conductivity, then the sensors will be positioned and attached over the muscle belly of the right trunk muscles (shown in table 2). The wireless EMG signals are transmitted to the EMG acquisition software via the electrodes pasted on the muscle belly, and then the raw EMG signals are processed and analysed using the EMG software (EMGworks Analysis and MATLAB) at a sampling frequency of $2000 \mathrm{~Hz}$, with band-pass filtering at 15-500 Hz. Each muscle's EMG data will be high-pass filtered, low-pass filtered and rectified to calculate the linear envelope describing muscle activation. EMG data were measured for $5 \mathrm{~s}$. After discarding the first and last second, the root-mean-squared, integrated EMG values and the mean power frequency and the median frequency of the three-second surface electromyography (sEMG) signals will be calculated. ${ }^{39} 40$ All muscle EMG characteristics will be assessed at 0,4 and 8 weeks during the study.

\section{Muscle onset times determination}

When the assessment of onset latency of the trunk muscles, subjects will be asked to perform rapid arm raises while quiet standing, which creates internal perturbations to the trunk and requires APAs. One electrode is placed over the right anterior deltoid $(\mathrm{AD})$ as the prime mover. Other electrodes placements sites of the trunk muscles are shown in table 2. Participants will be instructed to stand relaxed with their feet shoulder-width apart and arms by their sides, then they will be told to flex their left shoulder as fast as possible to the level of 90 degrees with the fullextended elbow. After familiarisation, five repetitions of left shoulder flexion trials will be recorded with a random 5-10 s interval. ${ }^{41}{ }^{42}$ Participants will be instructed to 'relax and breathe' while standing between each trial. All of the trunk muscle onset latency will be calculated as the difference between absolute trunk muscle onset times and the $\mathrm{AD}$ onset time. Muscle activation occurred between 200 $\mathrm{ms}$ before $\mathrm{AD}$ onset and $50 \mathrm{~ms}$ after is regarded as anticipatory. The average muscle onset time for the five repetitions of each trunk muscle will be calculated and formed the basis for data analysis. ${ }^{42}$

\section{RUSI on muscle thickness}

Images of the external oblique (EO)/internal oblique (IO)/TrA and LM will be acquired with a portable ultrasound machine in B-mode (SonoSite M-Turbo, Seattle, USA) with a 6-13 MHz linear-array transducer (for abdominal muscles) or 2-5 MHz curvilinear-array transducer (for lumber muscles), automatically adjusted by the scanning depth. To avoid affecting the muscle morphology, the examiner must be careful not to compress the skin with the transducer. The positions of the transducer, the same as the monitoring sites, are shown in table 2. To avoid the influence of respiration, images will be captured at the end of exhalation. All muscle thicknesses will be measured at 0,4 and 8 weeks during the study. ${ }^{4344}$ Muscle activation ratio=contraction thickness/relax thickness. Preferential activation ratio of $\operatorname{Tr} \mathrm{A}=\operatorname{Tr} \mathrm{A}$ contracted/ $(\mathrm{Tr} \mathrm{A}+\mathrm{IO}+\mathrm{EO})$ contracted-TrA rest/(TrA+IO+EO) rest. A higher value of the preferential contraction ratio indicates a relatively greater change in the contraction 
thickness of the TrA, whereas a lower value represents a relatively greater change in the contraction thickness of the EO and IO muscles.

\section{Data management}

All study data including the paper-based documents and electronic data will be stored at the Sun Yat-sen University for 15 years after the completion of the trial. All documents that contain participants' personal information will be identified by code number and stored separately. Only researchers involved in this study can be available to the confidential documents.

\section{Monitoring}

Because the study is not a drug trial and the sponsor or funder has no access to the raw data, a data monitoring committee will not be formed and there is no planned trial audit. Besides, there are no interim analyses and stopping guidelines due to the very low risks of adverse events and other unintended effects.

\section{Statistical analysis}

All of the statistical analyses will be based on the intentionto-treat principle and the Shapiro-Wilk test will be used to examine the normality of data distribution. Descriptive statistics including the number and proportions for categorical variables and means and SD for continuous variables will be recorded and displayed. Data are collected and analysed centrally after evaluation completion. Statistical analysis will be conducted using the SPSS V.22.0 software (IBM). Baseline demographics will be examined by descriptive statistics. Sphericity assumption will be identified by the test, and the differences of all the variables in each group will be compared using the repeated measures analyses of variance to examine intervention effects (dependent variables), with the group (SG and $\mathrm{CG})$ as between-subject variable and time $(0,4,8$ weeks and 6 months of follow-up) as the within-subject variable. If a significant interactive effect of time and group exists, the post-hoc tests for multiple comparisons with Bonferroni adjustments will be adopted. The significance level is set at a priori alpha level of 0.05 for all of these tests.

\section{Patient and public involvement}

Patients and the public were not involved in the development of this study protocol. Patients presenting for treatment to our clinic or being recruited from the internet who satisfied the inclusion criteria will be recruited for the proposed randomised controlled trial (RCT). All subjects included will receive related treatments for free. If needed, trial participants will be able to obtain their individual results from the trial after the completion of the study.

\section{DISCUSSION}

The primary aim of the present study is to examine the effect of the RUSI-based CSE in individuals with CNLBP. The pain intensity of LBP patients is assessed with VAS, the degree of disability is evaluated by ODI, and the quality of life is assessed with the SF-12. We hypothesise that CSE monitored by real-time biofeedback ultrasound will be superior compared with the CG for reducing pain, improving functional capacity as well as physical and mental health aspects.

Besides, the postural stability is examined with $\mathrm{COP}$ ways parameters, and the trunk position sense is represented by TRE. The effectiveness of postural control depends on the interaction between the neural and musculoskeletal systems, which is a suitable indicator for evaluating the function of the sensorimotor system. Previous studies have demonstrated that there are impaired postural control mechanisms, such as reduced postural strategy variability, increased COP sways, and difficulty in equilibrium maintenance after perturbation in subjects with CNLBP compared with healthy individuals. ${ }^{45}{ }^{46}$ In the present study, we expect that CSE with real-time biofeedback which targeted specific core muscles can improve the posture stability and position sense of the trunk.

Then, the trunk muscle onset latency in CNLBP individuals is evaluated based on the measurement of APAs during a postural task. During a rapid voluntary limb movement, the period before and up to $50 \mathrm{~ms}$ after the onset of the prime mover is defined as the anticipatory time window. ${ }^{47}$ Some studies indicated that compared with healthy controls, trunk muscle onsets are delayed in people with CLBP, which cannot be classified as an anticipatory or feedforward response. ${ }^{48} 49$ In our study, we speculate that RUSI-based core stabilising exercise will be useful to modify the delayed muscle onsets in CNLBP patients.

Finally, the trunk muscle activities are recorded by sEMG and the muscle thickness changes are measured with RUSI. Previous researches have reported that the imbalance of the trunk muscle force may lead to kinetic instability of the spine, while the weakness of local muscles, such as LM and TrA, may suboptimally load the passive tissues of the spine, contributing to the development and recurrence of LBP. Besides, the compensation activity of the superficial muscles during the process of training affects the effectiveness of CSE. Thus, muscles will be monitored under real-time biofeedback techniques to guarantee the correct training to facilitate selective activity and contraction of the TrA independently off the superficial muscles, which can be more beneficial than global exercise programmes on the restoration of deep muscles activation and strength.

Altogether, this is the first attempt to systematically investigate the efficiency of the RUSI-based core stabilising exercise in individuals with CNLBP. This trial will provide a quantitative analysis of the deep and superficial trunk muscle contraction and performance concerning changes in pain and functional status. It will provide randomised trial evidence of the clinical effectiveness of implementing RUSI biofeedback during CSE, which could potentially be a cost-effective method in clinical rehabilitation. 


\section{Ethics and dissemination}

This protocol and informed consent has been approved by the Institutional Research Ethics Committee of the First Affiliated Hospital, Sun Yat-sen University (Approval number: [2020] 254-1). Participants will be informed of the study objectives, its risks and benefits, and must sign the informed consent before the study begins. Any protocol amendments will be detailed in the trial registration. Besides, the study findings will be submitted to scientific meetings and will also be published in peerreviewed journals.

\section{Trial status}

This trial is currently recruiting participants and will require 6-8 months to complete all follow-up assessments.

Acknowledgements We would like to acknowledge the patients and staff at the First Affiliated Hospital, Sun Yat-sen University for their involvement piloting the intervention.

Contributors SLin and CW conceived of the study. BZ, SLiu and YZ have made substantial contributions to the design and conduction of the statistical analysis. BZ, SLiu and CW reviewed the manuscript critically, all authors contributed to the refinement of the study protocol and approved the final manuscript.

Funding The authors have not declared a specific grant for this research from any funding agency in the public, commercial or not-for-profit sectors.

Competing interests None declared.

Patient and public involvement Patients and/or the public were not involved in the design, or conduct, or reporting, or dissemination plans of this research.

Patient consent for publication Consent obtained directly from patient(s).

Provenance and peer review Not commissioned; externally peer reviewed.

Open access This is an open access article distributed in accordance with the Creative Commons Attribution Non Commercial (CC BY-NC 4.0) license, which permits others to distribute, remix, adapt, build upon this work non-commercially, and license their derivative works on different terms, provided the original work is properly cited, appropriate credit is given, any changes made indicated, and the use is non-commercial. See: http://creativecommons.org/licenses/by-nc/4.0/.

ORCID iD

Shanshan Lin http://orcid.org/0000-0002-0817-9098

\section{REFERENCES}

1 Violante FS, Mattioli S, Bonfiglioli R. Low-back pain. Handb Clin Neurol 2015;131:397-410.

2 Meucci RD, Fassa AG, Faria NMX. Prevalence of chronic low back pain: systematic review. Rev Saude Publica 2015;49:1.

3 Rosenfeld SB, Schroeder K, Watkins-Castillo SI. The economic burden of musculoskeletal disease in children and adolescents in the United States. J Pediatr Orthop 2018;38:e230-6.

4 Zusman M. Belief reinforcement: one reason why costs for low back pain have not decreased. J Multidiscip Healthc 2013;6:197-204.

5 Hicks GE, Sions JM, Velasco TO, et al. Trunk muscle training augmented with neuromuscular electrical stimulation appears to improve function in older adults with chronic low back pain: a randomized preliminary trial. Clin J Pain 2016;32:898-906.

6 Chou R, Deyo R, Friedly J, et al. Nonpharmacologic therapies for low back pain: a systematic review for an American College of physicians clinical practice guideline. Ann Intern Med 2017;166:493-505.

7 Furlan AD, Giraldo M, Baskwill A. Massage for low-back pain. Cochrane Database Syst Rev 2015;9:CD001929.

8 Liu L, Skinner M, McDonough S, et al. Acupuncture for low back pain: an overview of systematic reviews. Evid Based Complement Alternat Med 2015;2015:1-18.

9 Panjabi MM. The stabilizing system of the spine. part II. neutral zone and instability hypothesis. J Spinal Disord 1992;5:390-7.

10 Stevens VK, Coorevits PL, Bouche KG, et al. The influence of specific training on trunk muscle recruitment patterns in healthy subjects during stabilization exercises. Man Ther 2007;12:271-9.
11 Shamsi M, Sarrafzadeh J, Jamshidi A, et al. The effect of core stability and general exercise on abdominal muscle thickness in nonspecific chronic low back pain using ultrasound imaging. Physiother Theory Pract 2016;32:277-83.

12 Vasseljen O, Unsgaard-Tøndel M, Westad C, et al. Effect of core stability exercises on feed-forward activation of deep abdominal muscles in chronic low back pain: a randomized controlled trial. Spine 2012;37:1101-8.

13 Wallwork TL, Stanton WR, Freke M, et al. The effect of chronic low back pain on size and contraction of the lumbar multifidus muscle. Man Ther 2009;14:496-500.

14 Cresswell AG, Oddsson L, Thorstensson A. The influence of sudden perturbations on trunk muscle activity and intra-abdominal pressure while standing. Exp Brain Res 1994;98:336-41.

15 Coulombe BJ, Games KE, Neil ER, et al. Core stability exercise versus general exercise for chronic low back pain. J Athl Train 2017;52:71-2.

16 Smith BE, Littlewood C, May S. An update of stabilisation exercises for low back pain: a systematic review with meta-analysis. BMC Musculoskelet Disord 2014;15:416.

17 Wilson A, Hides JA, Blizzard L, et al. Measuring ultrasound images of abdominal and lumbar multifidus muscles in older adults: a reliability study. Man Ther 2016;23:114-9.

18 Djordjevic O, Djordjevic A, Konstantinovic L. Interrater and intrarater reliability of transverse abdominal and lumbar multifidus muscle thickness in subjects with and without low back pain. J Orthop Sports Phys Ther 2014;44:979-88.

19 Hibbs AE, Thompson KG, French DN, et al. Peak and average rectified EMG measures: which method of data reduction should be used for assessing core training exercises? J Electromyogr Kinesiol 2011;21:102-11.

20 Xiao J, Sun J, Gao J, et al. The activity of surface electromyographic signal of selected muscles during classic rehabilitation exercise. Rehabil Res Pract 2016;2016:1-9.

21 Hodges PW, Moseley GL. Pain and motor control of the lumbopelvic region: effect and possible mechanisms. J Electromyogr Kinesiol 2003;13:361-70.

22 Jeong J, Kim K, Kim J. Age related analysis of ultrasound images of normal skeletal muscle. J Korean Acad Univ Trained Phys Ther 2006;13:47-53.

23 Zheng Y, Ke S, Lin C, et al. Effect of core stability training monitored by rehabilitative ultrasound image and surface electromyogram in local core muscles of healthy people. Pain Res Manag 2019;2019:1-8.

24 Chan A-W, Tetzlaff JM, Gøtzsche PC, et al. Spirit 2013 explanation and elaboration: guidance for protocols of clinical trials. BMJ 2013;346:e7586.

25 Ma K, Zhuang Z-G, Wang L, et al. The Chinese association for the study of pain (CASP): consensus on the assessment and management of chronic nonspecific low back pain. Pain Research and Management 2019;2019:1-14.

26 Schulz KF, Chalmers I, Hayes RJ, et al. Empirical evidence of bias. Dimensions of methodological quality associated with estimates of treatment effects in controlled trials. JAMA 1995;273:408-12.

27 Puntumetakul R, Areeudomwong P, Emasithi A, et al. Effect of 10 -week core stabilization exercise training and detraining on painrelated outcomes in patients with clinical lumbar instability. Patient Prefer Adherence 2013;7:1189-99.

28 Lee N-G, Jung J-H, You JS-H, et al. Novel augmented ADIM training using ultrasound imaging and electromyography in adults with core instability. J Back Musculoskelet Rehabil 2011;24:233-40.

29 Konrad P. ABC of EMG - a practical introduction to kinesiological electromyography. 1 edn. Scottsdale, AZ, USA: Noraxon INC, 2005.

30 Park H-ju, Oh D-won, Kim S-yeop. Effects of integrating hip movements into bridge exercises on electromyographic activities of selected trunk muscles in healthy individuals. Man Ther 2014;19:246-51.

31 Martí-Salvador M, Hidalgo-Moreno L, Doménech-Fernández J, et al. Osteopathic manipulative treatment including specific diaphragm techniques improves pain and disability in chronic nonspecific low back pain: a randomized trial. Arch Phys Med Rehabil 2018;99:1720-9.

32 Ware J, Kosinski M, Keller SD. A 12-Item short-form health survey: construction of scales and preliminary tests of reliability and validity. Med Care 1996;34:220-33.

33 Wettstein M, Eich W, Bieber C, et al. Pain intensity, disability, and quality of life in patients with chronic low back pain: does age matter? Pain Med 2019;20:464-75.

34 Chen Z, Shen Z, Ye X, et al. Association between foot posture asymmetry and static stability in patients with knee osteoarthritis: a case-control study. Biomed Res Int 2020;2020:1-8. 
35 Pinsault N, Vuillerme N. Test-retest reliability of centre of foot pressure measures to assess postural control during unperturbed stance. Med Eng Phys 2009;31:276-86.

36 Taglietti M, Dela Bela LF, Dias JM, et al. Postural sway, balance confidence, and fear of falling in women with knee osteoarthritis in comparison to matched controls. Pm R 2017;9:774-80.

37 Goldberg A, Hernandez ME, Alexander NB. Trunk repositioning errors are increased in balance-impaired older adults. J Gerontol A Biol Sci Med Sci 2005;60:1310-4.

38 Toprak Celenay S, Mete O, Coban O, et al. Trunk position sense, postural stability, and spine posture in fibromyalgia. Rheumatol Int 2019;39:2087-94.

39 Qie S, Li W, Li X, et al. Electromyography activities in patients with lower lumbar disc herniation. J Back Musculoskelet Rehabil 2020;33:589-96.

40 Poitras I, Bielmann M, Campeau-Lecours A, et al. Validity of wearable sensors at the shoulder joint: combining wireless electromyography sensors and inertial measurement units to perform physical workplace assessments. Sensors 2019;19:1885.

41 Knox MF, Chipchase LS, Schabrun SM, et al. Improved compensatory postural adjustments of the deep abdominals following exercise in people with chronic low back pain. $J$ Electromyogr Kinesiol 2017;37:117-24.

42 Jacobs JV, Henry SM, Nagle KJ. People with chronic low back pain exhibit decreased variability in the timing of their anticipatory postural adjustments. Behav Neurosci 2009;123:455-8.
43 Kliziene I, Sipaviciene S, Klizas S, et al. Effects of core stability exercises on multifidus muscles in healthy women and women with chronic low-back pain. J Back Musculoskelet Rehabil 2015;28:841-7.

44 Lee J-Y, Lee D-Y. The effect of therapeutic abdominal drawingin maneuver using ultrasonography on lateral abdominal muscle thickness and balance. J Back Musculoskelet Rehabil 2018;31:1139-43.

45 Azadinia F, Ebrahimi-Takamjani I, Kamyab M. Immediate effects of lumbosacral orthosis on postural stability in patients with low back pain: a preliminary study. Arch Bone Jt Surg 2019;7:360-6.

46 Abbasi S, Rojhani-Shirazi Z, Shokri E, et al. The effect of Kinesio taping on postural control in subjects with non-specific chronic low back pain. J Bodyw Mov Ther 2018;22:487-92.

47 Knox MF, Chipchase LS, Schabrun SM, et al. Anticipatory and compensatory postural adjustments in people with low back pain: a systematic review and meta-analysis. Spine J 2018;18:1934-49.

48 Massé-Alarie H, Flamand VH, Moffet $\mathrm{H}$, et al. Corticomotor control of deep abdominal muscles in chronic low back pain and anticipatory postural adjustments. Exp Brain Res 2012;218:99-109.

49 Hemmati L, Piroozi S, Rojhani-Shirazi Z. Effect of dual tasking on anticipatory and compensatory postural adjustments in response to external perturbations in individuals with nonspecific chronic low back pain: electromyographic analysis. J Back Musculoskelet Rehabil 2018;31:489-97. 